\title{
Successful surgical repair of a sternum cleft using composite mesh: A case report and new technical note
}

\author{
N N M Razafimanjato, ${ }^{1} \mathrm{MD} ;$ G O Tsiambanizafy, ${ }^{1} \mathrm{MD} ; \mathrm{T}$ D N Ravelomihary, ${ }^{1} \mathrm{MD} ; \mathrm{H} \mathrm{J} \mathrm{L} \mathrm{Rakotovao,}{ }^{1} \mathrm{MD} ; \mathrm{F}$ A Hunald, ${ }^{2} \mathrm{MD}$ \\ ${ }^{1}$ Division of Thoracic Surgery, Department of Surgery, Faculty of Medicine, University of Antananarivo and Teaching Hospital of Joseph Ravoahangy \\ Andrianavalona, Antananarivo, Madagascar \\ ${ }^{2}$ Division of Pediatrics Surgery, Department of Surgery, Faculty of Medicine, University of Antananarivo and Teaching Hospital of Joseph Ravoahangy \\ Andrianavalona, Antananarivo, Madagascar
}

Corresponding author: N N M Razafimanjato (razafesteban@yahoo.fr)

\begin{abstract}
Sternal clefts are infrequent congenital malformations, particularly in their complete presentation. There are less than 100 descriptions of these defects published in the literature worldwide. We report a clinical case of lower sternal cleft associated with congenital laparoschisis in a 2-year-old boy. Surgery was performed because of recurrent pneumopathy and the risk of cardiorespiratory decompensation in the midterm. A semi-resorbable prosthesis was used for sternal closure. We have not observed any complications with this sternal closure system in our patient. This approach is easy, safe, effective and not harmful to a child's growth.

Keywords. bifid sternum; chest wall deformity; congenital abnormality; laparoschisis; pentalogy of Cantrell syndrome; sternal cleft.
\end{abstract}

Afr J Thoracic Crit Care Med 2021;27(2):66-69. https://doi.org/10.7196/AJTCCM.2021.v27i2.103

Sternal clefts are a rare idiopathic chest wall deformity caused by a defect in the sternum's congenital fusion process that can be complete or partial. ${ }^{[1-2]}$ In the literature, some sternal malformations are said to be major (compromising health) and non-viable (incompatible with survival), and they are associated with 'ectopia cordis' observed as part of a malformities named pentalogy of Cantrell. ${ }^{[2]}$ However, bifid sternum is usually not associated with large structural cardiac abnormalities but may be associated with trophic skin patch. ${ }^{[2]}$ Usually asymptomatic in the neonatal period (except for a paradoxical median thoracic swelling), it can lead to dyspnoea, cough, recurrent respiratory infections and an increased risk of traumatic injury to the heart, lungs and major vessels if it is not treated. Our patient presented with an inferior sternal cleft and upper median laparoschisis. The surgical operation consisted of the primary closure of the defect. Herein, we report a 2-year-old boy with bifid sternum beyond the neonatal period who underwent early successful primary closure using semi-resorbable composite mesh with a multidisciplinary surgical approach between anaesthesiologist, thoracic and paediatric surgeon in our centre.

\section{Case}

A 2-year-old boy with recurrent respiratory infection and an anterior chest wall swelling was referred by the paediatrician. The child was delivered via normal vaginal birth at 36 weeks of amenorrhea, eutrophic with no mention of recurrent urinary tract infection in the mother during the pregnancy period. The obstetrical ultrasounds were uneventful. Interrogation did not reveal any parental consanguinity, no height and weight deficit and vaccination status was up to date. The parents did not report cyanosis, dyspnoea, or repeated respiratory infections in the neonatal period. On clinical examination, the child presented with thoracoabdominal balanced breathing without respiratory difficulty. Observation and palpation diagnosed a sternal cleft. Physical examination revealed an enlargement of the caudal portion of the sternum and a laparoschisis responsible for a xyphoumbilical midline eventration that worsened with crying episodes (Fig. 1). The rest of the somatic examination was without abnormality including no associated visible malformation and a normal cardiac auscultation. The cardiac ultrasound-Doppler showed a 'situs

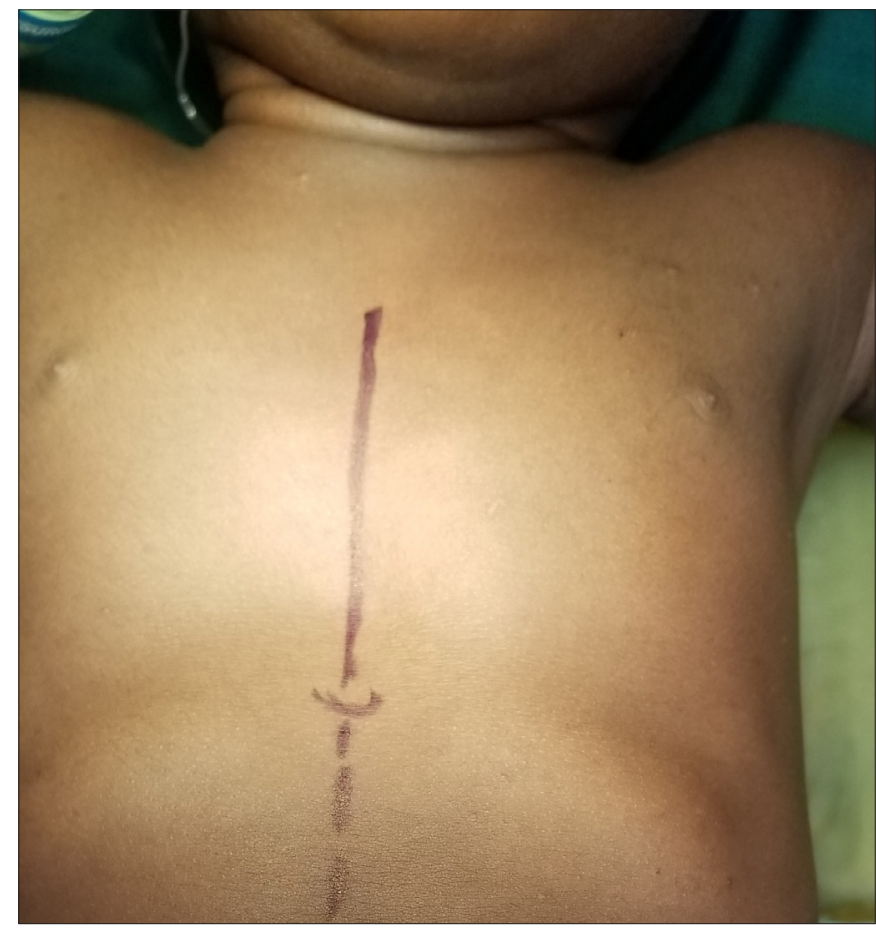

Fig. 1. Demonstration of the paradoxical movement of the lower chest wall 
solitus' without any notable cardiovascular abnormality. The chest computed tomography (CT) scan in axial sections with 3D parietal reconstruction showed the presence of small, rounded, hypodense and discontinuous formations corresponding to the ossification points in the upper part of the sternal edges and a sternal diastasis in the lower part (Fig. 2). The rest of the biological investigations revealed no abnormalities and the child's karyotype analysis had not been investigated due to the limited financial status of the family. We made a diagnosis of lower bifid sternum associated with congenital laparoschisis in a 2-year-old male child who was completely asymptomatic and without any other associated visceral malformation.

\section{Technical note}

Primary closure of the defect was necessary given the risks of recurrent pneumopathy and long-term cardio-respiratory decompensation. The operative procedure consisted of two operative steps, bone and muscular rapprochement with a composite mesh. It was performed on the patient in a supine position under general anaesthesia with ventilation via a classic orotracheal intubation. Prior to the incision, the correct anatomical landmarks (jugulum and xiphoid) had to be identified. We then undertook a
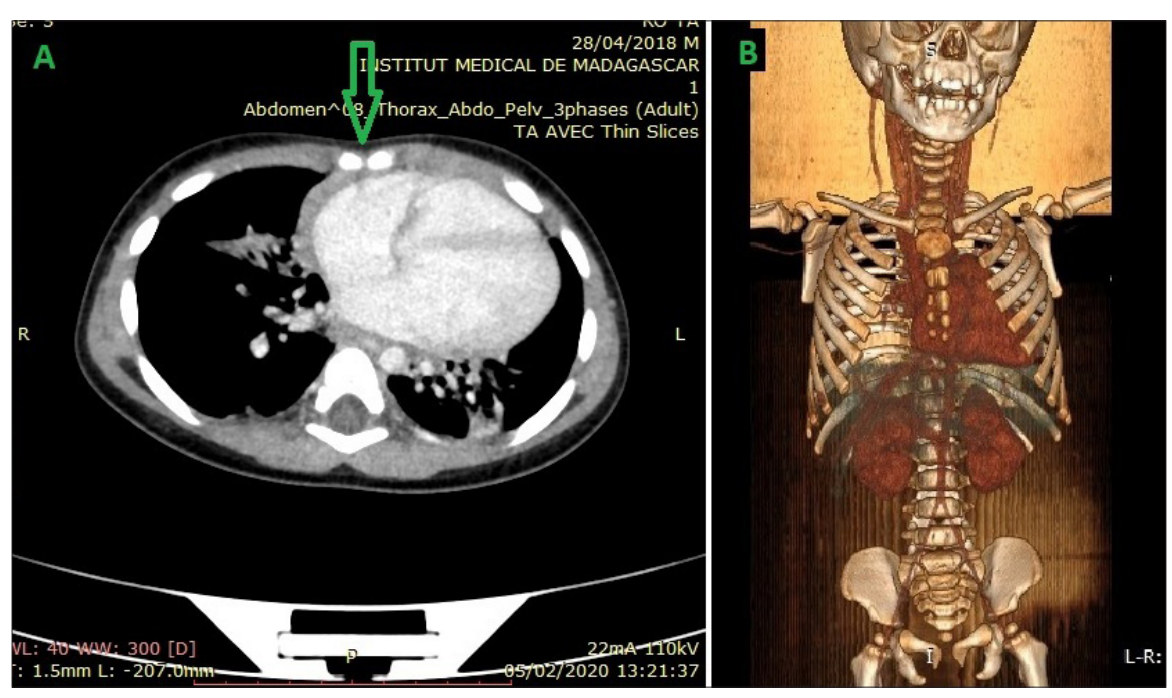

Fig. 2. Chest axial computed tomography (CT) scan (A) demonstrates lack offusion of the sternal margins, and (B) $3 D$ reconstruction of the CT scan of the chest showing the edges of the lateral sternal bards.
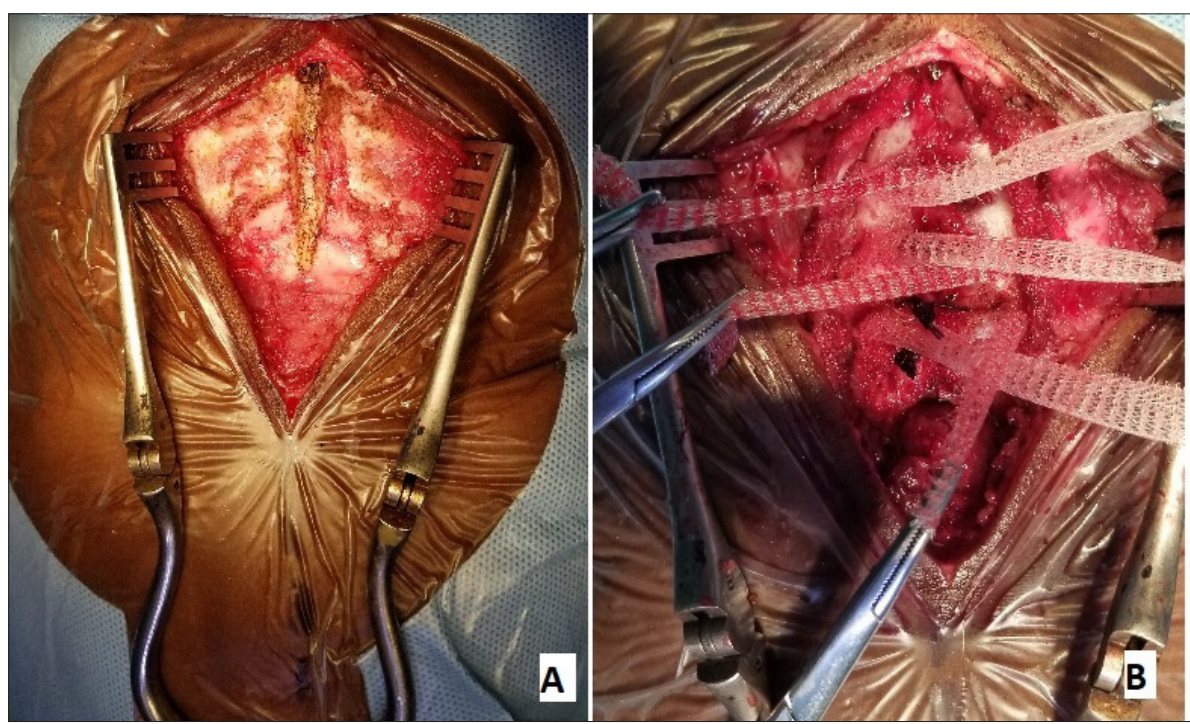

median skin incision and a subcutaneous cauterisation from the fossa jugularis to the xyphoidis, followed by a layer-by-layer dissection down to the sternum to reach the sternal cleft. The sternal bars were dissected free and isolated under the periosteum from the insertion of pectoralis major muscles anteriorly, and endothoracic fascia posteriorly (Fig. 3A). The inferior sternal portion was resected as an inverted V-shape. The pre-cut prosthesis (Parietex) was inserted behind the sternum and then unrolled. Each of the tips of the prosthesis were passed through the intercostal spaces (Fig. 3B), joined together and fixed with interrupted sutures without traction on the anterior surface body of the sternum (Fig. 3C). The prosthesis compensated and bridged the gap between the two sternal bars (Fig. 4). In a second operative step, the diastasis of the rectus abdominis muscles was reduced by separate stitches of PDS II (poly-pdioxanone) 3/0 and the pectoral muscles were brought together with VICRYL 3/0. Retrosternal drainage was placed and the subcutaneous plane was sutured with VICRYL 4/0. There were no postoperative complications. The patient tolerated the sternal closure well without any hemodynamic instability or change in the ventilator parameters. The postoperative follow-up was uneventful. The drain was removed on day 4 post operation and the patient was discharged 7 days after repair. The result was satisfactory in the medium and long term with good cicatrisation and adequate positioning of the sternal bars

Fig. 3. Intraoperative view of the reconstruction steps. Dissection of the anterioperiostem of the sternal bars which will be pulled medially and posteriorly (A), (B) composite mesh used to reinforce the sternum, and (C) view of the completed repair. 


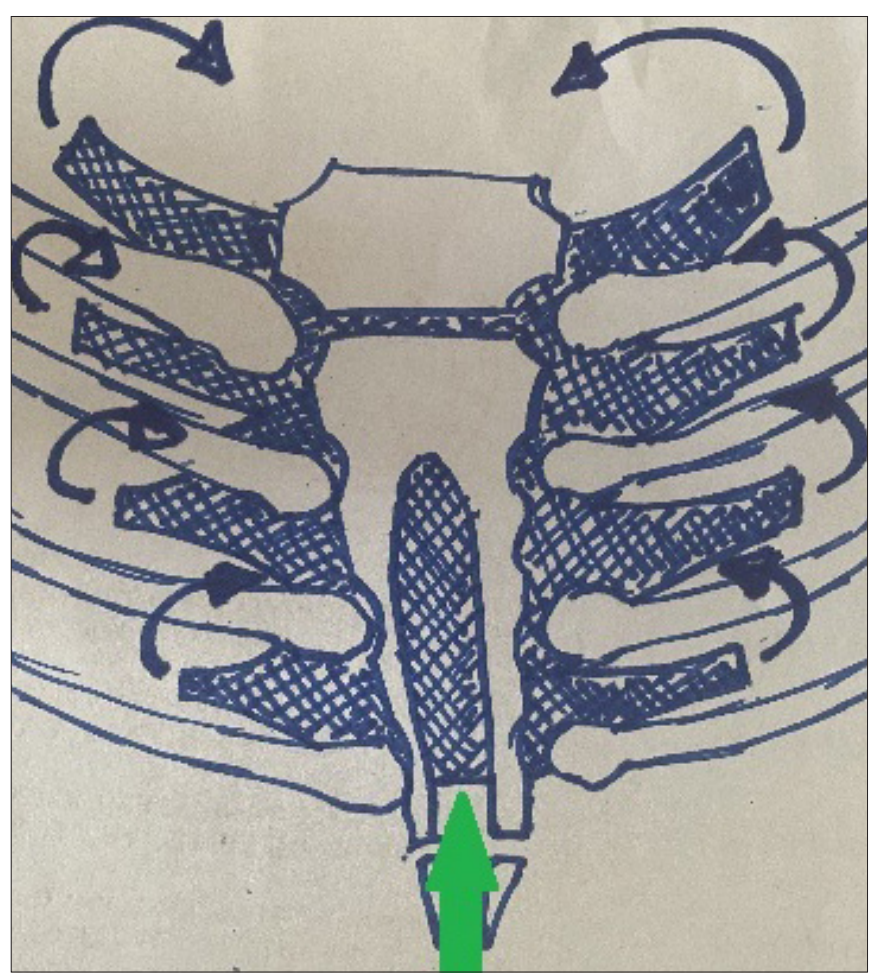

Fig. 4. Closing of the gap between the two sternal bars behind the sternum by the prosthesis (green arrow).

giving a good aesthetic appearance. The patient is now 4 years old and in excellent condition.

\section{Discussion}

The sternal fissure is a defect in the fusion of the sternum at an early stage of development of the fetus. ${ }^{[3]}$ It is a rare malformation, particularly in the form of an isolated defect that was first reported in 1740 by London. ${ }^{[3]}$ The first surgical correction was reported by Lannelongue in 1888 , but the first successful repair was published by Burton in 1947. ${ }^{[1]}$ Multiple embryological studies of the sternum have attributed the sternal cleft to a defect in fusion of the two sternal bands from the lateral plate mesoderm, which normally occurs around the ninth week of intrauterine life in the craniocaudal direction. ${ }^{[1,3-6]}$ The cleft may involve the manubrium and upper sternebrae, the lower sternum saving the xiphoid process, but also the entire sternum. Therefore, sternal clefts are classified as incomplete and partial forms, which can be superior or inferior. ${ }^{[4,5]}$ According to Ravitch ${ }^{[7]}$ classification of sternal clefts may be in the form of:

- Complete bifid sternum with or without ectopia cordis or herniation of pericardium known as Cantrell pentalogy (congenital sternal cleft, omphalocele, anterior diaphragmatic hernia, ectopia cordis, and a variety of intracardiac defects).

- Fusion at only upper or lower parts of sternal plates (isolated sternal defects).

- Incomplete fusion giving rise to a hole in the sternum.

We observed an association between the partial sternal cleft combined with a median xypho-umbilical laparoschisis. It should be noted that this association is infrequent. Only two other similar cases, to our knowledge, have been reported in the literature. ${ }^{[8,9]}$ The most common defect is the partial superior form, which affects the upper sternum and the manubrium with normal fusion of the lower part. ${ }^{[5,6]}$ Inferior defects are extremely rare and usually associated with complex syndromes like Cantrell pentalogy. ${ }^{[4,5]}$ None of this pentalogy was found in our patient. The aetiopathology of sternal clefts remains unclear. Haque ${ }^{[10]}$ identified familial cases in Saudi Arabia suggesting an autosomal recessive pathology. Chest wall development studies have suggested the implication of the Hoxb-4 gene and have been inconclusively associated it with defects in mice. ${ }^{[11]}$ Other experiments have demonstrated that perturbations of GSK-3 $\beta$ (glycogen synthase kinase-3 $\beta$ ) resulted in alterations of the para-axial mesoderm. ${ }^{[12]}$ Other hypotheses have been suggested, such as abuse of alcohol consumption, nutritional deficiencies and hypovitaminosis (methylcobalamin, riboflavin) during pregnancy but have not been proven. ${ }^{[3,8]}$ There is no reported justification for the predominantly female prevalence of the condition. ${ }^{[3-5]}$ However, studies by Gorlin et $a l .{ }^{[13]}$ and Heron et al. ${ }^{[14]}$ showed a predominance of females when the sternal cleft is associated with a bandlike scar from the umbilicus and diastasis recti.

A bifid or sternum cleft is a rare congenital anomaly generally diagnosed as asymptomatic at birth. ${ }^{[2,4-5]}$ Prenatal diagnosis of isolated sternal cleft is very difficult. ${ }^{[4]}$ However, Yuksel et al., ${ }^{[15]}$ diagnosed an intrauterine sternal cleft with the help of ultrasonography. In the neonatal period, the diagnosis of sternal cleft is easily done by inspection and palpation. ${ }^{[6]}$ Imaging based on conventional radiology, multidetector CT and magnetic resonance show the verticalisation of the clavicles, dehiscence of sternal bars, which is more important when the patient is seen at a later stage, and associated with malformations. CT is considered the best technique for studying sternal anatomy, offering the possibility to perform $3 \mathrm{D}$ evaluation, which can help the surgeon in surgical repair of the sternal cleft. ${ }^{[5-6]}$

Historically, Lannelongue ${ }^{[1]}$ carried out the first surgical repair of a complete sternal cleft in 1988. Burton ${ }^{[8]}$ published two cases of successful surgical repair by implanting a cartilage graft in the defect in the bone in 1947. Subsequently, Maier and Bortone ${ }^{[16]}$ carried out direct closure of two hemi-sternums in infants who were 6 weeks old and this technique has become the reference. ${ }^{[8]}$ Whether symptomatic or not, the sternal cleft requires surgical correction in the new born, when the flexibility of the chest wall is maximal and compression of underlying structures is minimal to restore bony protection to the mediastinal structures, establish normal intrathoracic pressure relationships, improve respiratory dynamics and for aesthetic reasons. ${ }^{[2,4,6]}$ In the literature, repairs performed after the age of 3 months have always required more supportive postoperative care, with a higher incidence of cardiac complication. ${ }^{[5,8]}$ Beyond one year, autologous grafts (costal cartilage, parietal skull, tibial periosteum) or prosthetic materials such as Marlex mesh, Teflon mesh, silicone elastomer and acrylic are required. ${ }^{[5-}$ ${ }^{6]}$ Moreover, studies have suggested that it is always preferable to use autogenous tissue for sternal cleft repair and to avoid the use of prosthetic materials, recognising the risk of infection and the negative impact of inert material on the patient's development. ${ }^{[1,4,8]}$

Despite the risk of infection described in the literature, the use of prosthesis in sternal cleft repair has an advantage in preventing an increase in intrathoracic pressure such as explained in our technical note. ${ }^{[17]}$ In addition, this surgical approach avoids major surgical 
dissection and the use of autogenous or bone bank rib reconstruction, which presents a potential risk of aseptic or septic necrosis of the bone ${ }^{[18]}$ What sets Parietex apart from other prosthetic material is the density it can achieve from relatively little pressure. Most other prosthetics take thousands of pounds of pressure to squeeze them into soft shapes. The other benefits of using this type of prosthesis are its flexibility and conformity to the anatomy once implanted. The resorbable hydrophilic film promotes rapid parietal integration to provide a temporary barrier and minimise visceral attachment to mesh. ${ }^{[19]}$ The nonabsorbable component provides stability and bridge the gap between the two sternal bars while avoiding direct approximation of both sternal halves and to prevent increasing intrathoracic pressure. It allows fast and complete tissue in-growth on one side for efficient reinforcement. A study by Casha et al. ${ }^{[20]}$ evaluated and quantified the rigidity of sternotomy fixation using a mechanical model through which six different fixation techniques were tested: figure of 8 , straight, Ethibond, repair, Sternaband and multitwist, and showed that Sternaband with its flat ribbon shape, similar to the cutting of the prosthesis in our technique, is more resistant to cutting than wires. The force imparted by the lateral part of the closure can help bring direct approximation of both sternal halves, providing stability. To the best of our knowledge, our case is the first reparation of sternal cleft using composite mesh and since this defect is so rare, we don't have a vast experience on this subject. However, this surgical approach with mesh prosthesis can be indicated in many types of sternal clefts (superior sternal cleft, subtotal sternal cleft, total sternal cleft, inferior sternal cleft and median sternal cleft) to guarantee favourable long term results with reference to the stature growth, reproducibility, and adaptsbility to all surgical centres that train in general surgery.

\section{Conclusion}

The neonatal period is the best period for surgical correction of sternal clefts due to the elasticity of the sternum and minimal compression of the underlying structures. All infants with recurrent pulmonary infection should be referred to surgeons for an early investigation to detect malformation of either the lungs or the chest wall to avoid delayed surgical repair. A considerable variety of sternal cleft repair procedures have been reported in the literature. However, our surgical procedure is safe and relatively easy, preventing any impact on the child's stature and postural development.

Declaration. None.

Acknowledgements. None.

Author contributions. NNMR, TDNR and GOT drafted the manuscript and were the surgeons responsible for treating the patient. FAH and
HJLR edited the manuscript. All authors approved the final manuscript for publication.

Funding. None.

Conflicts of interest. None.

1. Demircan M, Gü3rbüz N, Karaman A. A bifid sternum case underwent the earliest repair in the literature. İnönü Üniversitesi Tip Fakültesi Dergisi 2010;12(4):253-255.

2. Aronson LA, Martin DP. Anaesthesia and bifid sternum repair in an infant. J Clin Anesth 2013;25(4):324-326. https://doi.org/10.1016/j.jclinane.2012.11.010.

3. Chellaoui $\mathrm{M}$, Chat $\mathrm{L}$, Achaabal $\mathrm{F}$, et al. Fissure sternale congénitale totale à propos d'un cas. Médecine du Maghreb 2001;90(3);16-18.

4. Das SK, Jana PK, Bairagya TD, et al. Bifid sternum. Lung India 2012;29(1):73. https://doi.org/10.4103\%2F0970-2113.92370.

5. Manenti G, Bozzi A, Ferrazzoli V, et al. Bifid sternum in a young woman Multimodality imaging features. Radiol Case Rep 2017;12(4):672-677. https://doi. org/10.1016\%2Fj.radcr.2017.06.005.

6. Dòmini M, Cupaioli M, Rossi F, et al. Bifid sternum: Neonatal surgical treatment. Ann Thorac Surg 2000;69(1):267-269. https://doi.org/10.1016/S0003-4975(99)01206-0.

7. Ravitch MM. Congenital deformities of the chest wall and their operative correction. Philadelphia: Saunders, 1977:23-25.

8. Beurdeley M, Gauthier T, Bedu A, et al. Fente sternale: Intérêt d'une prise en charge chirurgicale néonatale. Arch Pédiatr 2010;17(10):1477-1479. https://doi. org/10.1016/j.arcped.2010.07.012.

9. Hebra A, Davidoff A, O’Neill Jr JA. Neonatal sternal cleft associated with an extrathoracic cystic mass. J Pediatr Surg 1997;32:627-630. https://doi.org/10.1016/ s0022-3468(97)90724-7.

10. Haque KN. Congenital absence of sternum. Arch Childhood 1979;54:905-906.

11. Forzano F. Midline raphe, sternal cleft and other midline abnormalities: A new dominant syndrome? Am J Med Genet 2005;135A:9e12. https://doi.org/10.1002/ ajmg.a.30682.

12. Liu KJ, Arron JR, Stankunas K, et al. Chemical rescue of cleft palate and midline defects in conditional GSK-3 $\beta$ mice. Nature 2007;446(7131):79-82. https://doi. org/10.1038/nature05557.

13. Gorlin RJ, Kantaputra P, Aughton DJ, et al. Marked female predilection in some syndromes associated with facial hemangiomas. Am J Med Genet 1994;52:130-135. https://doi.org/10.1002/ajmg.1320520203.

14. Heron D, Lyonnet S, Iserin L, et al. Sternal cleft: Case report and review of a series of nine patients. Am J Med Genet 1995;59:154-156. https://doi.org/10.1002/ ajmg.1320590207.

15. Yuksel M, Kuru P, Ermerak NO, et al. Intrauterine diagnosed sternal cleft patient and her management. J Vis Surg 2016;2:48. https://doi.org/10.21037\%2Fjovs.2016.03.04.

16. Torre M, Rapuzzi G, Carlucci M, et al. Phenotypic spectrum and management of sternal cleft: Literature review and presentation of a new series. Eur J Cardiothorac Surg 2012;41(1):4-9. https://doi.org/10.1016\%2Fj.ejcts.2011.05.049.

17. Kuwata T, Park S, Sakano I, Kuwata K. Congenital superior sternal cleft repair using our modified Sally technique: A case report. J Pediatr Surg Case Reports. 2014;2(12),544-547. https://doi.org/10.1016/j.epsc.2014.11.001.

18. Hoffman E. Surgical correction of bifid sternum: Using marlex mesh. Arch Surg 1965;90(1):76-80. https://doi.org/10.1001/archsurg.1965.01320070078017.

19. Doctor HG. Evaluation of various prosthetic materials and newer meshes for hernia repairs. J Min Access Surg 2006;2:110-116. https://doi.org/10.4103/09729941.27721.

20. Casha AR, Yang L, Kay PH, Saleh, Cooper GJ. A biomechanical study of median sternotomy closure techniques. Eur J Cardiothoracic Surg 1999;15(3):365-369. https://doi.org/10.1016/s1010-7940(99)00014-7.

Accepted 9 March 2021. 